\title{
Comparison of Sapphire and Germanium Fibers for Erbium : Yag Lithotripsy
}

\author{
Ho Lee, Ji-Wook Yoon, and Young-Dae Jung \\ School of Mechanical Engineering, Kyungpook National University, Daegu 702-701, Korea \\ JeeHyun Kim* \\ Department of Computer Engineering, Kyungpook National University, Daegu 702-701, Korea \\ Robert T. Ryan \\ Division of Urology, University of Texas Health Science Center, San Antonio, Texas 78229, USA \\ Joel M.H. Teichman \\ Division of Urology, St. Paul's Hospital, Department of Urologic Sciences, University of British \\ Columbia, \#C307-1081 Burrard St., Vancouver, B.C. V6Z 1Y6, Canada \\ A.J. Welch \\ Department of Biomedical Engineering, The University of Texas at Austin, 1 University Station, \\ \#C0800, Austin, Texas 78712, USA
}

(Received October 28, 2008 : revised December 9, 2008 : accepted December 10, 2008)

\begin{abstract}
We studied the sapphire and germanium fibers to determine which optical fiber best transmits Erbium:YAG laser for intracorporeal lithotripsy. Human calculi were ablated with an Erbium:YAG laser in contact mode using two fibers. Optical outputs at the distal end of fibers were measured before and after laser lithotripsy. Upon the irradiation on the calculus with the $50 \mathrm{~mJ}$ and 100 $\mathrm{mJ}$ pulse energy, the output energy at the distal end of germanium fiber declined to approximately $50 \%$ of the input energy. For the sapphire fiber, the output energy at the distal end remained unchanged with $100 \mathrm{~mJ}$ input energy; however the output energy had dropped to 50\% for 200 mJ input energy. In order to examine how the types of target tissue affect the fiber damage, the sapphire fiber was tested for the irradiation on soft tissue and water as well. No energy decline was observed during soft tissue and water irradiation. We also characterized ablation craters with both optical fibers. Both fibers produced similar craters on calculi in terms of depth and diameter. Sapphire fibers are better suited than germanium fibers for Erbium:YAG lithotripsy in terms of the fiber damage.
\end{abstract}

Keywords: Laser lithotripsy, Erbium:YAG, Germanium fiber, Sapphire fiber

OCIS codes : (170.0170) Medical optics and biotechnology; (060.2270) Fiber characterization; (140.3500) Lasers, Erbium; (170.1020) Ablation of tissue; (170.7230) Urology.

\section{INTRODUCTION}

Intracorporeal laser lithotripsy has been established as one of the standard treatment modalities for human

*Corresponding author: jeehk@knu.ac.kr calculi fragmentation since the first development of the $\mathrm{CO}_{2}$ laser lithotripsy [1-6]. Due to its strong absorption by calculi of multiple compositions, the Erbium:YAG laser has been tested for the next generation lithotripter recently [7-9]. The Erbium:YAG laser operates at a wavelength of $2.94 \mu \mathrm{m}$ and a pulse duration of $250-300$ 
usec. Erbium:YAG lithotripsy fragments calculi by a photothermal mechanism [10]. Although this lithotripsy mechanism is similar to Holmium:YAG lithotripsy, Erbium:YAG is more efficient than Holmium:YAG lithotripsy [7-9]. Erbium:YAG wavelength is highly absorbed by water [11]. Thus, the flexible low-OH silica fibers used in Holmium:YAG applications absorb Erbium: YAG energy when used for Erbium:YAG transmission, causing reduced optical energy output. Currently, the optical fibers available for transmission of Erbium:YAG energy are composed of sapphire, germanium, zirconium fluoride, or hollow waveguide. To be employed as an endoscopic delivery system, the fiber needs to be biocompatible, flexible, and robust to tolerate the strong lasertissue interaction at the distal end of the fiber.

Hybrid fibers have been proposed as an endoscopic delivery system for the laser lithotripsy. Fried et al. employed a hybrid germanium-silica/germanium-sapphire fiber to protect the germanium fiber tip from a damage induced by the strong pulse tissue interaction [12-14]. A similar approach is being pursued using a hollow waveguide with a sealing cap [15]. Although these hybrid techniques still do not deliver pulse energy high enough for the efficient lithotripsy, further improvement is expected from an advanced assembly and protective cap.

In spite of the extensive studies on the hybrid-fiber system, the performance of the bare sapphire and germanium fibers for the Erbium:YAG lithotripsy has received a paucity of study [16]. In order to provide the reference for the hybrid fiber systems, we studied the sapphire and germanium fibers to determine how well they transmit Erbium:YAG optical energy for lithotripsy applications.

\section{EXPERIMENTAL RESULTS}

Human struvite calculi ( $>95 \%$ pure magnesium ammonium phosphate hexohydrate) were cut with a dental diamond band saw to create a flat surface for laser irradiation. Calculi were hydrated in deionized water for a minimum of 24 hours. A experimental Erbium:YAG laser (Schwartz 1-2-3, Orlando, FL, wavelength: $=2.94 \mu \mathrm{m}$, pulse duration $=275 \mu \mathrm{sec}$ ) was coupled into either a 425 $\mu \mathrm{m}$ diameter sapphire(Photran, Amherst, NH, USA, core diameter : $425 \mu \mathrm{m}$, no cladding, Transmission per meter : 80\%, effective NA : 0.12) or $450 \mu \mathrm{m}$ diameter germanium optical fibers(Infrared Fiber Systems, Silver Spring, MD, USA, core diameter : $450 \mu \mathrm{m}$, Transmission Loss at $2.94: 0.70 \mathrm{~dB} / \mathrm{m}$, Max acceptance NA : 0.25) using a $\mathrm{CaF}_{2}$ lens as shown in Fig. 1. Erbium:YAG laser was operated in the free running mode with the pulse repetition rate of $2 \mathrm{~Hz}$. The unstable initial ten pulses were blocked using a mechanical shutter in conjunction with a shutter controller. The laser and shutter controller were synchronized using a pulse generator. Fibers were

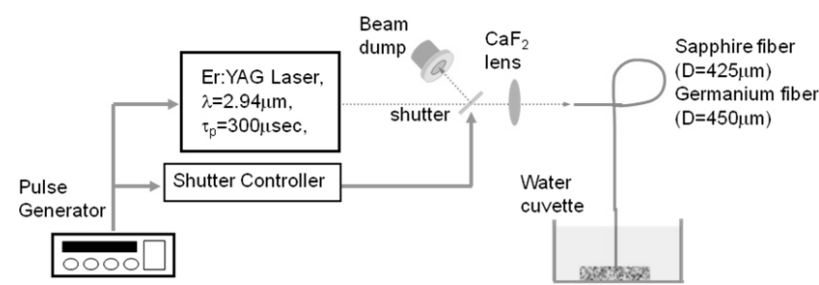

FIG. 1. The schematic diagram of the experimental setup. A free running Erbium:YAG laser was coupled into sapphire and germanium fibers, and human calculus was irradiated with both fibers in contact mode.

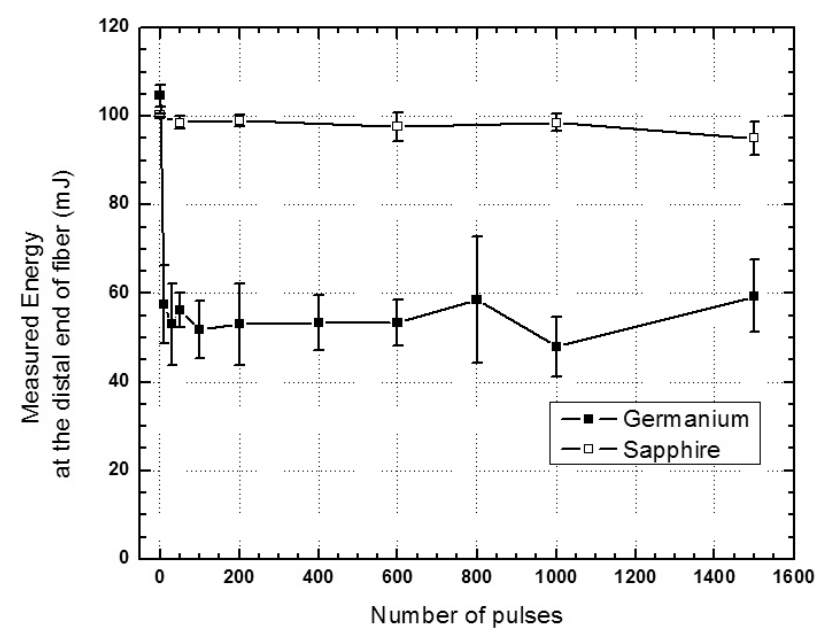

FIG. 2. Output pulse energy at the distal end of the fiber vs. the number of irradiation pulses. Input pulse energy to the proximal end was set to about $100 \mathrm{~mJ}$. The irradiation was made on the struvite calculus in contact mode. A total of five measurements were made and the error bar indicates the standard deviation of the measurements.

polished prior to use and the fiber tip verified by microscopy to be smooth and free of visible defects. Stones were placed in a water cuvette and optical fibers were placed in contact with the stone surface. Irradiation was performed at a right angle orientation $\left(0^{\circ}\right.$ laser incidence) between the fiber and stone surface. The energy output from the tip of the optical fibers was measured using an energy detector (Molectron, Portland, OR) at various intervals before and after lithotripsy to assess the damage at the distal end of the fiber damage.

The initial output energy at the distal end of the fiber was set to about $100 \mathrm{~mJ}$ then we ablated stone with both fibers. The output pulse energy after the irradiation of $10,30,50,100,200,400,600,800,1000,1500$ pulses was measured with the energy detector. The measurement has been repeated five times for both fibers. In case of the Germanium fiber, the pulse energy had declined to $50 \% \sim 60 \%$ of the initial pulse energy during the first 50-100 pulses and then relatively stable thereafter as shown in Fig. 2. In contrast, there was no significant 


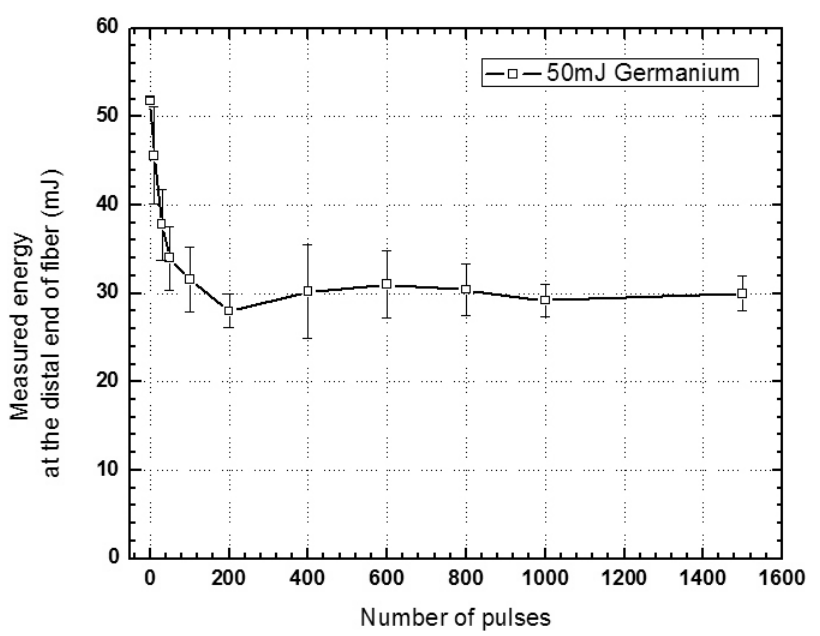

FIG. 3. Output pulse energy at the distal end of the Germanium fiber vs. the number of irradiation pulses. Input pulse energy to the proximal end was set to about 50 mJ. The irradiation was made on the struvite calculus in contact mode. A total of five measurements were made and the error bar indicates the standard deviation of the measurements.

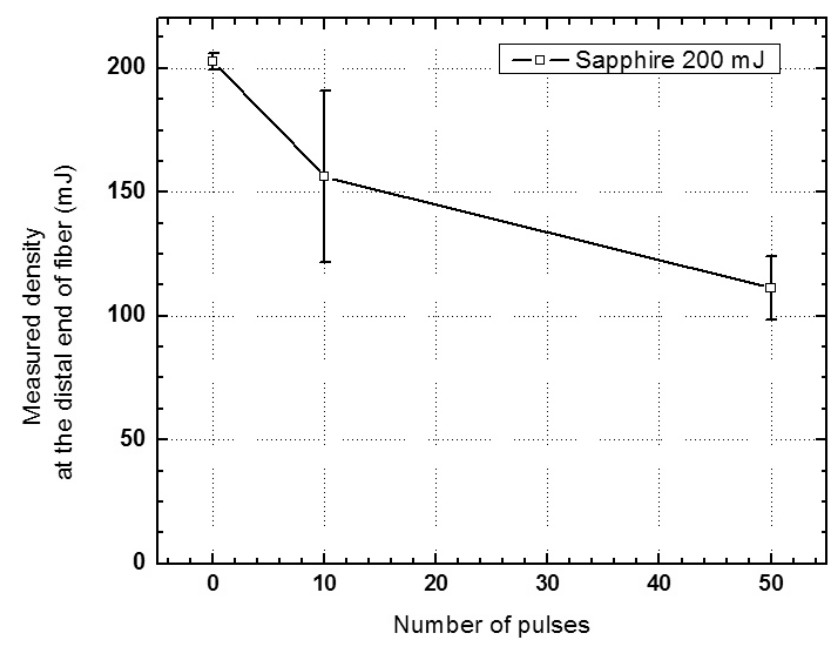

FIG. 4. Output pulse energy at the distal end of the Sapphire fiber vs. the number of irradiation pulses. Input pulse energy to the proximal end was set to about 200 mJ. The irradiation was made on the struvite calculus in contact mode. A total of five measurements were made and the error bar indicates the standard deviation of the measurements.

decline in the measured pulse energy outputs for the sapphire fiber using $100 \mathrm{~mJ}$ pulse energy. The pulse energy had been maintained above $90 \mathrm{~mJ}$ regardless of the number of pulses. At all measures (other than at 0 pulses), the measured optical energy outputs were greater for sapphire vs. germanium fibers.

Based on the preliminary experiments showing damage to the germanium fiber at $100 \mathrm{~mJ}$ pulse energy, we repeated the calculus irradiation with the germanium

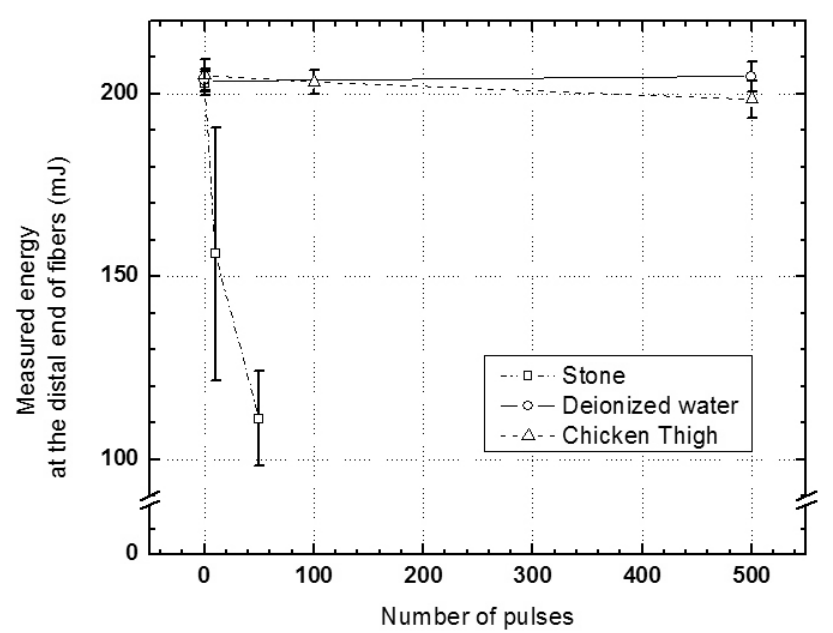

FIG. 5. Output pulse energy at the distal end of the Sapphire fiber vs. the number of irradiation pulses. Input pulse energy to the proximal end was set to about 200 mJ. The irradiation was made on the struvite calculus, into the distilled water and on chicken thigh in contact mode. A total of five measurements were made and the error bar indicates the standard deviation of the measurements.

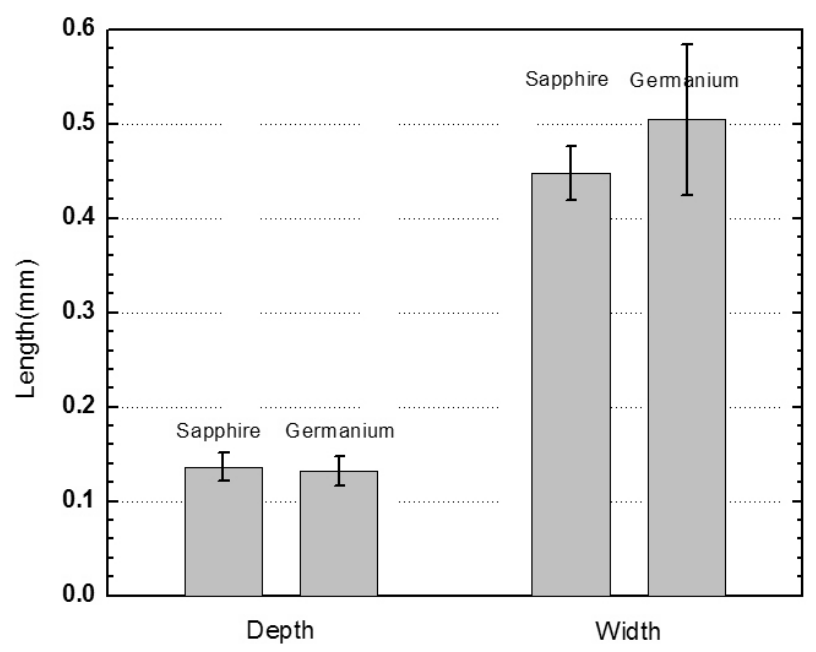

FIG. 6. Caparison of the crater depth and width. Craters were produced upon the irradiation of Erbium:YAG laser through either the Sapphire or Germanium fiber.

fiber using $50 \mathrm{~mJ}$ pulse energy for 1500 pulses. The measurement has been repeated five times. As we observed with the $100 \mathrm{~mJ}$ input energy, the pulse energy had drop to 50\% 60\% during the first 50-100 pulses and then relatively stable thereafter as shown in Fig. 3.

As the preliminary experiments showed the minimal damage to the sapphire fiber, we repeated calculus irradiation with the sapphire fiber using $200 \mathrm{~mJ}$ pulse energy and measured energy outputs. The output pulse energy after the irradiation of 10 and 50 pulses was measured with the energy detector. The measurement 
has been repeated five times. At this energy level, the sapphire fiber damaged easily, the output energy dropped to $50 \sim 60 \%$ of initial energy within the first 50 pulses.

In order to compare the hard and soft tissue interaction with sapphire fiber, we irradiated $200 \mathrm{~mJ}$ laser energy to the deionized water and the muscle tissue of chicken thigh which was placed in the deionized water. We irradiated up to 500 pulses to both target and energy was measured using the energy meter after 100 and 500 pulses. A total of five measurements were made. In contrast to the hard tissue interaction, no significant energy decline was observed during the laser interaction with water and soft tissue. In case of the irradiation in still water, the measured energy outputs pre-irradiation versus after 500 pulses were $204 \pm 3 \mathrm{~mJ}$ vs. $205 \pm 4 \mathrm{~mJ}$, $\mathrm{p}=0.680$. In case of the chicken breast irradiation, the measured energy outputs were pre-irradiation $205 \pm 4$ $\mathrm{mJ}$, after 100 pulses $203 \pm 3 \mathrm{~mJ}$, versus after 500 pulses $198 \pm 5 \mathrm{~mJ}$.

The ablation craters produced with both optical fibers were characterized. We irradiated single craters with pulse energy of $50 \mathrm{~mJ}$ at multiple locations on the stone surface. Crater width and depth were assessed by optical coherence tomography. We used two-tailed unpaired t-test and analysis of variance for analyses. The widths of ablation craters for sapphire were smaller than germanium fibers, $448 \pm 28 \mu \mathrm{m}$ vs. $504 \pm 80 \mu \mathrm{m}, \mathrm{p}=0.056$, and the depths were not statistically different $136 \pm 15$ $\mu \mathrm{m}$ vs. $132 \pm 16 \mu \mathrm{m}, \mathrm{p}=0.509$, respectively.

\section{DISCUSSION AND CONCLUSION}

The sapphire fiber is better than the germanium fiber for Erbium:YAG transmission. During laser lithotripsy, the damage threshold of sapphire fiber was measured between $100 \mathrm{~mJ}$ and $200 \mathrm{~mJ}$, while the damage threshold of germanium fiber was measured below $50 \mathrm{~mJ}$. Sapphire has a higher damage threshold, with less diminution of optical energy output during lithotripsy compared to germanium. Both fibers appear to produce roughly equivalent ablation craters on struvite calculi. However, neither fiber is ideal and both still fall short of clinical utility.

The damage that occurs to optical fibers appears to be from the laser-stone interaction and not from Erbium transmission. There was no diminution of energy transmission using sapphire fibers in still water at $200 \mathrm{~mJ}$ pulse energy after 500 pulses. However, with stone irradiation, there was a dramatic and rapid diminution. We infer that rapid ejection of stone debris is a possible cause of fiber degradation[5,18]. The diminution in energy output cannot be attributed to cavitation bubbles or pressure transients, as irradiation in still water would have caused the greatest damage. With the irradiation of chicken breast, there was a trend of minimal energy diminution. Erbium:YAG irradiation may be useful for soft tissue applications where maximal ablation and minimal coagulation is desired, such as for ureteral or urethral strictures. However, both bare sapphire and germanium fibers appear to have limited clinical lithotripsy practical utility.

\section{ACKNOWLEDGMENT}

This work was supported by following grants: Fundamental Science Research Fund (R01-2007-000-2056-0), The Research and Development on Oriental Medicine Treatment Technology funded by the Ministry of Health, Welfare, and Family Affair(B080033) and the Korea Research Foundation Grant funded by the Korean Government (MOEHRD, Basic Research Promotion Fund), KRF-2008-314-C00149.

\section{REFERENCES}

[1] W. P. Mulvaney and C. W. Beck, "The laser beam in urology,” J. Urol., vol. 99, no. 1, pp. 112-115, 1968.

[2] S. P. Dretler, "Laser lithotripsy: A review of 20 years of research and clinical applications," Lasers Surg. Med., vol. 8, no. 4, pp. 341-356, 1988.

[3] K. F. Chan, T. J. Pfefer, J. M. H. Teichman and A. J. Welch, "A perspective on laser lithotripsy: The fragmentation processes," J. Endourology, vol. 15, no. 3, pp. 257-273, 2001.

[4] J. M. H. Teichman, G. J. Vassar, J. T. Bishoff and G. C. Bellman, "Holmium:YAG fragments are smaller than Lithoclast, pulsed dye, or electrohydraulic lithotripsy," J. Urol., vol. 159, no. 1, pp. 17-23, 1998.

[5] G. J. Vassar, J. M. H. Teichman and R. D. Glickman, "Holmium:YAG lithotripsy efficiency varies with energy density," J. Urol., vol. 160, no 2, pp. 471-476, 1998

[6] M. Sofer, J. D. Watterson, T. A. Wollin, L. Nott, H. Razvi and J. D. Denstedt, "Holmium:YAG laser lithotripsy for upper urinary tract calculi in 598 patients," J. Urol., vol. 167, no 1, pp. 31-4, 2002.

[7] J. M. H. Teichman, K. F. Chan, P. P. Cecconi, N. S. Corbin, A. D. Kamerer, R. D. Glickman and A. J. Welch, “Er:YAG versus Ho:YAG lithotripsy," J. Urol., vol. 165, no. 3, pp. 876-879, 2001.

[8] H. Lee, H. W. Kang, J. M. H. Teichman, J. Oh and A. J. Welch, "Urinary calculus fragmentation during Ho:YAG and Er:YAG lithotripsy," Lasers Surg. Med., vol. 38, no. 1, pp. 39-51, 2006.

[9] K. F. Chan, D. X. Hammer, B. Choi, A. J. Welch, J. M. H. Teichman, H. S. McGuff, H. Pratisto and E. D. Jansen, "Free electron laser lithotripsy threshold radiant exposures,” J. Endourology, vol. 14, no. 2, pp. 161-167, 2000 .

[10] K. F. Chan, H. Lee, J. M. H. Teichman, A. Kamerer, H. S. McGuff, G. Vargas and A. J. Welch, "Erbium:YAG laser lithotripsy mechanism,” J. Urol., vol. 168, no. 2, pp. 436-441, 2002. 
[11] G. M. Hale and M. R. Query, "Optical constants of water in the 200-nm to 200-um wavelength region," Appl. Opt., vol. 12, no. 3, pp. 555-563, 1973.

[12] C. A. Chaney, Y. Yang and N. M. Fried, "Hybrid germanium/silica optical fibers for endoscopic delivery of Erbium:YAG laser radiation," Lasers Surg. Med., vol. 34, no. 1, pp. 5-11, 2004.

[13] Y. Yang, C. A. Chaney and N. M. Fried, "Erbium:YAG laser lithotripsy using hybrid germanium/silica optical fibers," J. Endourology, vol. 18, no. 9, pp.830-835, 2004.

[14] T. J. Polletto, A. K. Ngo, A. Tchapyjnikov, K. Levin, D. Tran and N. M. Fried, "Comparison of germanium oxide fibers with silica and sapphire fiber tips for transmission of Erbium: YAG laser radiation," Lasers Surg.
Med., vol. 38, no. 8, pp. 787-791, 2006.

[15] K. Iwai, Y. W. Shi, K. Nito, Y. Matsuura, T. Kasai, M. Miyagi, S. Saito, Y. Arai, N. Ioritani, Y. Okagami, M. Nemec, J. Sulc, H. Jelinkova, M. Zavoral, O. Kohler and P. Drlik, "Erbium:YAG laser lithotripsy by use of a flexible hollow waveguide with an end-scaling cap," Appl. Opt., vol. 42, no. 13, pp. 2431-2435, 2003.

[16] H. Lee, T. R. Ryan, A. Lee, J. H. Teichman, A. J. Welch, "Feasibility study of Er:YAG lithotripsy," Lasers Surg. Med., vol. 15 (Suppl), pp. 12, 2003.

[17] S. S. Spore, J. M. H. Teichman, N. S. Corbin, P. C. Champion, E. D. Williamson and R. D. Glickman, "Holmium:YAG lithotripsy: optimal power settings," $J$. Endourol, vol. 13, no.8, pp. 559-566, 1999. 